\title{
A conceptual framework for reporting experience of physical activity in COPD
}

\author{
Enrico M. Clini ${ }^{1,2}$ and Laura Fregonese ${ }^{3}$
}

Affiliations: 'University of Modena Reggio Emilia, Dept of Medical and Surgical Sciences, Pavullo, Italy. ${ }^{2}$ Ospedale Villa Pineta, Pavullo, Italy. ${ }^{3}$ European Medicines Agency, London, UK.

Correspondence: Enrico M. Clini, University of Modena Reggio Emilia, Ospedale Villa Pineta, Via Gaiato 127. Pavullo, 41026 Italy. E-mail: enrico.cliniQunimore.it

0 @ERSpublications

PROs integrate patients' perspective into clinical/regulatory assessment; conceptual framework adds new information http://ow.ly/AqXrO

The importance of physical activity in chronic diseases is increasingly acknowledged. The amount of physical activity has been shown to predict mortality indirectly in patients suffering from multiple conditions [1], while physical inactivity is important predictor of the risk of hospital readmission, and associated with higher mortality in elderly [2] and in patients with chronic obstructive pulmonary disease (COPD) [3, 4]. However, even though physical activity represents one of the most intriguing extrapulmonary clinical aspects of patients suffering from COPD, there is little evidence that either pharmacological or nonpharmacological interventions (e.g. counselling and rehabilitation) can enhance and durably maintain increased levels of physical activity in COPD or, conversely, that an increase in physical activity would result in favourable outcomes relevant to COPD patients [5]. Methods quantifying physical activity objectively, such as portable and wearable devices to monitor daily activities, are available and will allow further research on physical activity in COPD, and on its relation to the progression of the disease and the response to treatment [6-8].

COPD is traditionally measured by lung function, mainly forced expiratory volume in $1 \mathrm{~s}$ (FEV1) and the FEV1/forced vital capacity ratio. FEV1 correlates poorly with important symptoms such as breathlessness and with the impact of the disease on daily life in COPD [9]; it is therefore important to complement lung function assessment with measures directly related to the patients' experience of the disease, such as patientreported outcomes (PROs), for a comprehensive evaluation of the disease. "PRO" is an umbrella term covering single and/or multidimensional measures of symptoms, health-related quality of life (HRQoL), health status, adherence to treatment, satisfaction, etc. [10]. The importance of PROs for regulatory purposes, according to the US Food Drug Administration (FDA), is based on: 1) some treatment effects are known only to the patient; 2) there is a desire to know the patients' perspective on the effectiveness of a treatment; and 3) systematic assessment of the patients' perspective may provide valuable information that can be lost when that perspective is filtered through a clinician's evaluation [11].

The article by DobBels et al. [12] in this issue of the European Respiratory Journal presents the initial results of the Innovative Medicines Initiative-funded PROactive project (www.proactiveCOPD.com), aimed at developing PROs capturing the dimension of physical activity in COPD, mainly for use clinical trials. The authors describe the methodological process leading to two new PRO instruments in stable patients with COPD, one for daily use and one for site visits. The three-step qualitative study (one-to-one interviews, focus groups and cognitive debriefing) included 116 patients from four different countries. Three main

The views expressed in this article are the personal views of the authors and should not be understood or quoted as being made on behalf of or reflecting the position of the European Medicines Agency or one of its committees or working parties.

Received: Aug 122014 | Accepted: Aug 122014

Conflict of interest: None declared.

Copyright @ERS 2014 
themes emerged: amount and impact of physical activity; symptoms experienced during physical activity; and need for adaptation to facilitate individuals' physical activity. Steps 2 and 3, based on focus groups with experts and cognitive debriefing with patients, resulted in two tools suitable for a five-point multiple response option (Likert's scale): a 30-item PRO daily instrument and a 34-item PRO 7-day recall instrument. The authors concluded that their qualitative study is able to "help to understand the experience of physical activity from the patients' perspective" [12]. Differently from other assessment tools already available, the method used here starts with building the conceptual framework of physical activity from the patients' perspective.

The creation of a conceptual framework for PROs is recommended by the FDA [11] and methodological guidance is further elaborated in documents from the International Society for Pharmacoeconomics and Outcomes Research (ISPOR) [13]. The involvement of patient inputs in concept elicitation and the confirmation of the framework by qualitative methods, such as the focus groups and one-to-one interviews performed, are recommended to support content validity [13]. In this view, the work of DoBBELS et al. [12] is an interesting example of translation into practice of the FDA and ISPOR recommendations. The resulting conceptual framework seems adequate, at this stage, to capture the relevant clinical concept. The authors "frame" subconcepts/domains and their relations (qualitative/conceptual and mathematical/ statistical) around the main concept (the aspect of health status that the PRO is intending to measure) of physical activity. For example, an item measuring breathlessness would be measuring the subconcept of dyspnoea (a "symptom" type of concept) within physical activity, and an item measuring walking up stairs would be related to physical functioning/level of activity within physical activity but would also most likely correlate with dyspnoea. From the perspective of capturing the concept of relevance, this innovative study guides the exploration of the wide field of PRO methodology in pulmonary diseases beyond COPD.

While the importance of PROs is acknowledged by regulators, only $23 \%$ of the 308 new substances and biologics authorised in the USA between 2000 and 2012 were granted PRO claims, and among these, the majority $(81 \%)$ were based on PROs of signs and symptoms (e.g. pain) followed, at large, by functioning and HRQoL [14]. A reason of such low rate of success was, among others, the overlap of many PRO concepts with those of functional end-points [15]. This raises the issue of how to estimate, in advance, the extent to which the inclusion of a new PRO measure, such as the one presented, can provide added value in the clinical trial setting; in other words, if and when the collection of a given PRO's data can make a difference to the study conclusions. The latter is linked, besides to the robustness of the conceptual framework, to the clinical significance of the concept measured. At present, there is no agreed-upon classification of concepts, and no concept of "ranking" or priority in COPD, and labelling of concepts varies among researchers. The concept of physical activity proposed by DobBels et al. [12] is extremely relevant to COPD; however, its changes in relation to treatment are not completely known. The capability of the proposed physical activity tool to capture response to treatment, its timing and the influence of or confounding by, for example, exacerbations, comorbidities, psychological status and motivation (the last two expected mostly on items related to amount of physical activity and need for adaptation) will have to be assessed in the context of the validation of use phase and in larger populations.

PROs in COPD can be measured with general and disease-specific instruments [16], some of which have been demonstrated to be reliable and sensitive to changes at the single-patient level following interventions [17]. From a regulatory perspective, PROs to be considered as end-points (primary, co-primary and secondary) are those with great validity in capturing group responses. The sample size of 116 patients giving inputs to the physical activity PROs would appear, in this context, quite small. Patients' inclusion was based on age, sex and severity according to Global Initiative for Chronic Obstructive Lung Disease (GOLD) stage. The selection of the inclusion criteria as well as of the suggested quota of patients fulfilling each criteria was performed during the 2009 European Respiratory Society Annual Congress by an ad hoc multidisciplinary expert panel discussing the key demographic, clinical and psychosocial phenotyping criteria of COPD and their measurement. Even though consensual, these criteria were subjectively chosen, and the study did not require balancing across the different centres. GOLD class II and III represent the majority of the population participating in the development of the conceptual framework proposed by DobBels et al. [12] (79 patients), reflecting, according to the authors, the most prevalent severity in clinical trials. Future studies in the pipeline of PROactive might clarify the applicability of the developed PRO to the whole COPD spectrum and, in particular, its ability to detect change in more severe populations, as a minimal clinically important difference might be difficult to detect using a physical activity tool mainly based on, for example, outdoor activities.

One obstacle in the regulatory use of PRO tools in COPD is the difficulty of balancing possible lower sensitivity to effects in specific populations and higher background noise of multidimensional PRO tools (e.g. measuring health status) versus potential lower generalisability of mono-conceptual tools in a disease as 
heterogeneous and multidimensional as COPD. For example, relevant symptom PROs such as dyspnoea are difficult to standardise for regulatory purposes [18]. Recent European Medicines Agency guidance on PROs in the field of oncology [10] emphasises the role that a PRO end-point is intended to play in a clinical trial. The development and validation of PROs present major limitations, in particular when researchers pool the results from different instruments with different constructs used in clinical trials [19]. Indeed, at the present state of knowledge, it is difficult to advice on the use of one versus another PRO tool for regulatory purposes in COPD. There is the need to create consensus among academia, industry and regulatory bodies so that chosen PRO measures are matched appropriately to specific treatment objectives in clinical trials, driven by the study aim, in combination with the domains of the instrument. For this purpose, it might be important to establish whether tools developed following the establishment of a conceptual framework have a different discriminative or evaluative profile than existing tools. It has been suggested that the lack of standardised and accepted PRO tools for assessing the different aspects of COPD can hinder accurate evaluation of the efficacy of new therapies [16]. However, treatments with better efficacy than the ones currently available would be useful for assessing the value of PROs in relation to treatment response in COPD, in addition to robust studies correlating the PRO with the different aspects of the natural history of the disease.

In conclusion, valid PROs are needed for integrating the patients' perspective into clinical, regulatory and quality of care assessment. Due to its complexity and heterogeneity, COPD can represent a model for other chronic, multifactorial diseases. Methodological approaches based on a conceptual framework such as that proposed by DOBBELs et al. [12] may result in new information useful for different purposes, provided that they are validated as "fit for purpose". As no size fits all in COPD, the use of each PRO tool will have to be carefully planned according to specific objectives.

\section{References}

Myers J, Prakash M, Froelicher V, et al. Exercise capacity and mortality among men referred for exercise testing. N Engl J Med 2002; 346: 793-801.

2 Yohannes AM, Baldwin RC, Connolly M. Mortality predictors in disabling chronic obstructive pulmonary disease in old age. Age Ageing 2002; 31: 137-140.

3 Garcia-Aymerich J, Farrero E, Félez MA, et al. Risk factors of readmission to hospital for a COPD exacerbation: a prospective study. Thorax 2003; 58: 100-105.

4 Waschki B, Kirsten A, Holz O, et al. Physical activity is the strongest predictor of all-cause mortality in patients with COPD. Chest 2011; 140: 331-342.

5 Watz H, Pitta F, Rochester C, et al. An official ERS statement on physical activity in chronic obstructive pulmonary disease. Eur Respir J 2014 [In press DOI: 10.1183/09031936.00046814].

6 Van Remoortel H, Raste Y, Louvaris Z, et al. Validity of six activity monitors in chronic obstructive pulmonary disease: a comparison with indirect calorimetry. PLoS One 2012; 7: e39198.

7 Rabinovich RA, Louvaris Z, Raste Y, et al. Validity of physical activity monitors during daily life in patients with COPD. Eur Respir J 2013; 42: 1205-1215.

8 Van Remoortel H, Hornikx M, Demeyer H, et al. Daily physical activity in subjects with newly diagnosed COPD. Thorax 2013; 68: 962-963.

9 Cazzola M, MacNee W, Martinez FJ, et al. Outcomes for COPD pharmacological trials: from lung function to biomarkers. Eur Respir J 2008; 31: 416-469.

10 European Medicines Agency Oncology Working Party. Reflection Paper on the use of patient reported outcome (PRO) measures in oncology studies. EMA/CHMP/292464/2014. www.ema.europa.eu/docs/en_GB/document_ library/Scientific_guideline/2014/06/WC500168852.pdf Date last accessed: July 2014. Date last updated: November 30, 2014.

11 US Dept of Health and Human Services, Food and Drug Administration, Center for Drug Evaluation and Research, et al. Guidance for industry: patient-reported outcome measures - use in medical product development to support labeling claims. www.fda.gov/downloads/Drugs/GuidanceComplianceRegulatoryInformation/Guidances/UCM193282. pdf Date last accessed: July 2014. Date last updated: December 2009.

12 Dobbels F, de Jong C, Drost E, et al. The PROactive innovative conceptual framework on physical activity. Eur Respir J 2014; 44: 1223-1233.

13 Rothman M, Burke L, Erickson P, et al. Use of existing patient-reported outcome (PRO) instruments and their modification: the ISPOR good research practices for evaluating and documenting content validity for the use of existing instruments and their modification. PRO Task Force Report. Value Health 2009; 603: 1075-1083.

14 Gnanasakthy A, Mordin M, Clark M, et al. Patient-reported outcomes a review of patient-reported outcome labels in the United States: 2006 to 2010. Value Health 2012; 15: 437-442.

15 Gnanasakthy A, Lewis S, Clark M, et al. Potential of patient-reported outcomes as nonprimary endpoints in clinical trials. Health Qual Life Outcomes 2013; 11: 83.

16 Jones P, Miravitlles M, van der Molen T, et al. Beyond FEV1 in COPD: a review of patient-reported outcomes and their measurement. Int J Chron Obstruct Pulmon Dis 2012; 7: 697-709.

17 Singer JP, Yusen RD. Defining patient-reported outcomes in chronic obstructive pulmonary disease: the patientcentered experience. Med Clin North Am 2012; 96: 767-787.

18 Frei A, Svarin A, Steurer-Stey C, et al. Self-efficacy instruments for patients with chronic diseases suffer from methodological limitations-a systematic review. Health Qual Life Outcomes 2009; 7: 86.

19 Gross NJ. Chronic obstructive pulmonary disease outcome measurements: what's important? What's useful? Proc Am Thorac Soc 2005; 2: 267-271. 